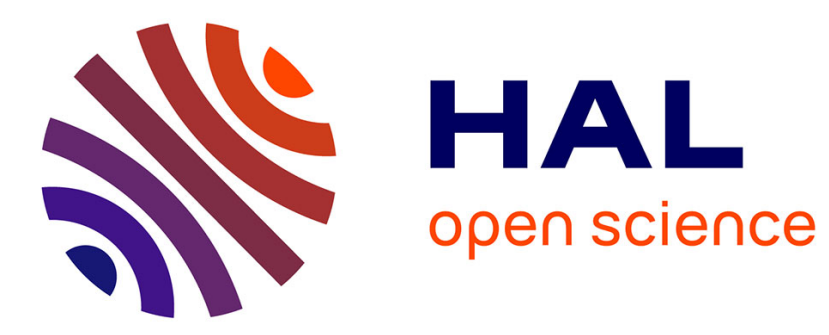

\title{
Deprit's reduction of the nodes revisited
}

\author{
Luigi Chierchia, Gabriella Pinzari
}

\section{To cite this version:}

Luigi Chierchia, Gabriella Pinzari. Deprit's reduction of the nodes revisited. Celestial Mechanics and Dynamical Astronomy, 2011, 109 (3), pp.285-301. 10.1007/s10569-010-9329-8 . hal-00609755

\section{HAL Id: hal-00609755 https://hal.science/hal-00609755}

Submitted on 20 Jul 2011

HAL is a multi-disciplinary open access archive for the deposit and dissemination of scientific research documents, whether they are published or not. The documents may come from teaching and research institutions in France or abroad, or from public or private research centers.
L'archive ouverte pluridisciplinaire HAL, est destinée au dépôt et à la diffusion de documents scientifiques de niveau recherche, publiés ou non, émanant des établissements d'enseignement et de recherche français ou étrangers, des laboratoires publics ou privés. 


\title{
Deprit's reduction of the nodes revisited*
}

\author{
Luigi Chierchia \\ Dipartimento di Matematica \\ Università "Roma Tre" \\ Largo S. L. Murialdo 1, I-00146 Roma (Italy) \\ luigi@mat.uniroma3.it \\ Gabriella Pinzari \\ Dipartimento di Matematica ed Applicazioni "R. Caccioppoli" \\ Università di Napoli "Federico II" \\ Monte Sant'Angelo - Via Cinthia I-80126 Napoli (Italy) \\ pinzari@mat.uniroma3.it
}

November 24, 2010

Revised version

\begin{abstract}
We revisit a set of symplectic variables introduced by Andre Deprit in [5], which allows for a complete symplectic reduction in rotation invariant Hamiltonian systems, generalizing to arbitrary dimension Jacobi's reduction of the nodes. In particular, we introduce an action-angle version of Deprit's variables, connected to the Delaunay variables, and give a new hierarchical proof of the symplectic character of Deprit's variables.
\end{abstract}

Keywords: Symplectic coordinates. Action-angle variables. Deprit reduction of the nodes. $\mathrm{N}-$ body problem. Planetary system.

MSC2000 numbers: 70H15, 70H33, 70F10, 70F15, 70E55, 34C20, 34D10

*Partially supported by European Research Council under F P 7 project "New connections between dynamical systems and Hamiltonian PDEs with small divisors phenomena". 


\section{Contents}

1 Deprit's reduction of the nodes 2

2 Proofs $\quad 10$

2.1 Case $n=2$ (three-body case) . . . . . . . . . . . . . . . . 11

2.2 Induction $(n \geq 2) \ldots \ldots \ldots \ldots$

$\begin{array}{ll}\text { A Deprit's energy-time variables } & 18\end{array}$

B Unfolding of Jacobi's reduction of the nodes 20

$\begin{array}{ll}\text { References } & 22\end{array}$

\section{Deprit's reduction of the nodes}

In 1983 Andre Deprit [5] introduced a new set of symplectic variables particularly suited to describe the phase space of Hamiltonian systems having rotational symmetries. The construction of Deprit may be viewed as a full extension to arbitrary numbers of degrees of freedom of Jacobi's reduction of the nodes for the three-body problem [6]. But in contrast with Jacobi's celebrated result, Deprit's variables seem to be not well known ${ }^{1}$ and often believed to be unpractical (as mentioned by Deprit himself [5, p. 194] or, e.g., in ${ }^{2}$ [7]). On the contrary, Deprit's variable may be very useful and can be effectively used (after a suitable "Poincaré regularization") to compute, for example, Birkhoff normal forms for the planetary $(1+n)$-body problem or to check KAM nondegeneracies; compare [4], [3].

Our presentation differs from that of Deprit in two respects. First we introduce action-angle variables (which are related to Delaunay's action-angle variables), while Deprit uses polar symplectic variables (compare (7) below); similarly to Delaunay's variables, our action-angle variables are particularly suited to describe planetary models. Secondly, we provide a different proof (presented in $\S 2$ ) of the symplectic character of the variables: such a proof is inductive on the number of dimensions and makes more transparent the hierarchical nature of Deprit's variables.

It has to be remarked that the action-angle version of Deprit's variables (as introduced below) may be defined only for osculating Keplerian orbits which lie on ellipses; on the other hand, Deprit is able to define his original variables on an open

\footnotetext{
${ }^{1}$ At the present date (September 2010) the MathSciNet database reports only one citation of Deprit's paper.

${ }^{2}$ In [7] a different formal procedure for the reduction of the angular momentum is worked out: After restricting to the vertical angular momentum manifold, the authors construct a set of symplectic variables perturbatively, through Taylor expansion, restoring the standard symplectic form at each step; the convergence of the formal power series thus obtained is not discussed.
} 
set of full measure of the global phase space. This is a minor technical point: using "energy-time" symplectic variables (i.e., giving up normalizations of the coordinates) one can easily generalize our symplectic construction to an open set of full measure of the global phase space; see Appendix A.

We conclude the paper (Appendix B) by comparing, in the $n=2$ case, with Jacobi's reduction: we show how, for $n=2$, Deprit's variables may be viewed as an "unfolding" of Jacobi's reduction, making more precise the statement that Deprit's variable extend to arbitrary dimensions Jacobi's reduction of the nodes.

More comments and remarks are made at the end of this section.

To define Deprit's variables on the phase space $\mathcal{P}^{6 n}$ (specified below), fix $2 n$ positive "mass parameters" $M_{i}, \bar{m}_{i}$ and consider the two-body Hamiltonians

$$
h_{i}\left(y^{(i)}, x^{(i)}\right):=\frac{\left|y^{(i)}\right|^{2}}{2 M_{i}}-\frac{M_{i} \bar{m}_{i}}{\left|x^{(i)}\right|}, \quad(1 \leq i \leq n)
$$

where $\left(y^{(i)}, x^{(i)}\right)$ are Cartesian variables in $\mathbb{R}^{3} \times \mathbb{R}_{*}^{3}:=\mathbb{R}^{3} \times\left(\mathbb{R}^{3} \backslash\{0\}\right)$, endowed with the standard symplectic form $\sum_{i=1}^{n} d y^{(i)} \wedge d x^{(i)}$. Assume that the Hamiltonian flow $\phi_{h_{i}}^{t}\left(y^{(i)}, x^{(i)}\right)$ evolves on Keplerian conics $\mathfrak{E}_{i}$ with eccentricity $e_{i} \neq 0$.

In our presentation we will focus on the case $h_{i}<0$ for all $i$ (corresponding to osculating ellipses), but the formulae can be easily extended to any energy, as explained in Appendix A below.

Let $a_{i}, P_{i}$ denote, respectively, the semimajor axis and the perihelion of the $i^{\text {th }}$ ellipse $\mathfrak{E}_{i}$ and let $\ell_{i}$ be mean anomaly of $x^{(i)}$ on $\mathfrak{E}_{i}$.

Define, now, $(n+1)$ nodes $\nu_{1}, \nu_{2}, \cdots, \nu_{n+1}$ as follows.

Put

$$
\mathrm{C}^{(i)}:=x^{(i)} \times y^{(i)}, \quad S^{(i)}:=\sum_{j=1}^{i} \mathrm{C}^{(j)} .
$$

In particular, $\mathrm{C}:=S^{(n)}=\sum_{1 \leq j \leq n} \mathrm{C}^{(j)}$ is the total angular momentum. Assuming that the couples of vectors $\left(S^{(\overline{i+1})}, \mathrm{C}^{(i+1)}\right),\left(\mathrm{C}, k^{(3)}\right)$ are independent, put

$$
\left\{\begin{array}{l}
\nu_{i+1}:=S^{(i+1)} \times \mathrm{C}^{(i+1)}, \quad 1 \leq i \leq n-1 \\
\nu_{1}:=\nu_{2} \\
\nu_{n+1}:=k^{(3)} \times \mathrm{C}=: \bar{\nu}
\end{array}\right.
$$

$\left(k^{(1)}, k^{(2)}, k^{(3)}\right)$ being the standard orthonormal triple in $\mathbb{R}^{3}$.

Notice that $\bar{\nu}$ is the node of the invariable plane (i.e., of the plane orthogonal to C) with the fixed $\left(k^{(1)}, k^{(2)}\right)$-plane; $\nu_{2}, \cdots, \nu_{n}$ are the nodes of the orbital planes (i.e., orthogonal to the $\mathrm{C}^{(i+1)}$ 's) with those orthogonal to the $S^{(i+1)}$ 's. For later convenience, the node of the orbital plane of $\mathrm{C}^{(1)}$ (with respect to $S^{(2)}$ ) is chosen 


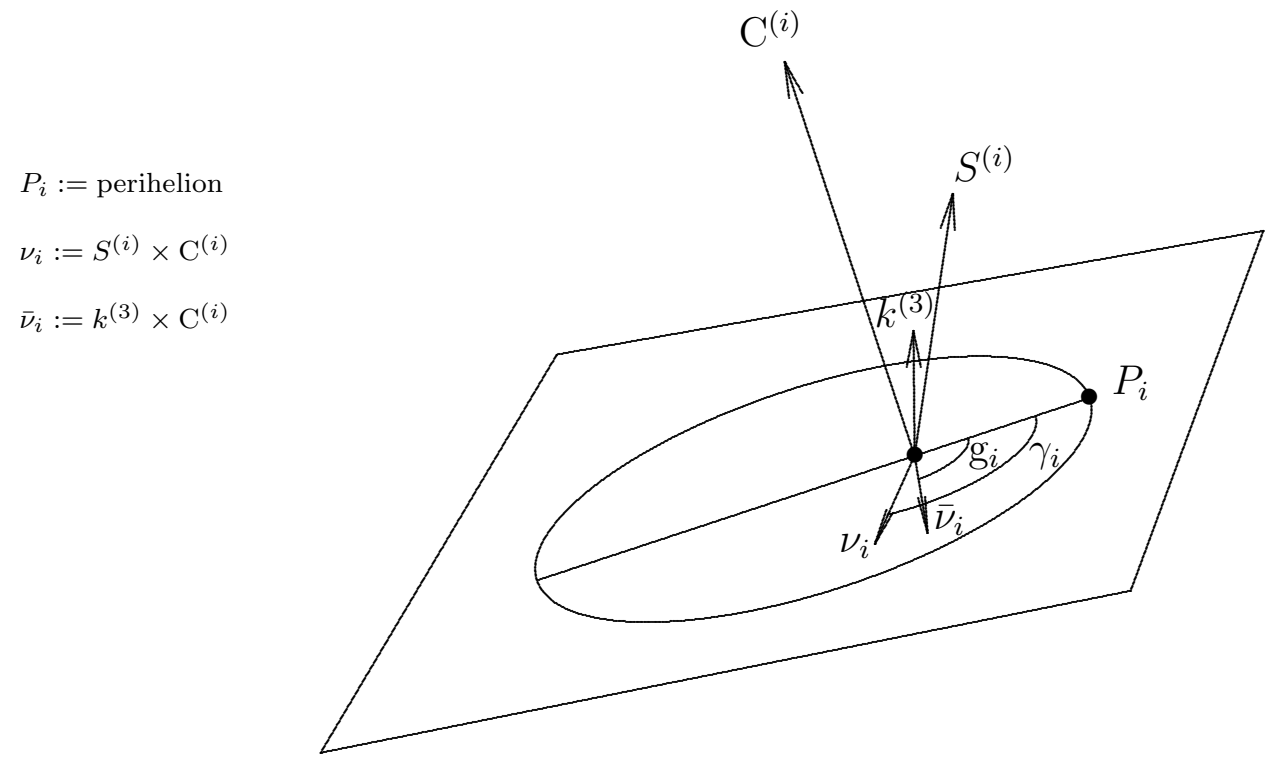

Figure 1: The angle $\gamma_{i}$

here $^{3}$ to be in the same direction of $\nu_{2}$, i.e., it is chosen to be $\nu_{1}:=-S^{(2)} \times \mathrm{C}^{(1)}=$ $-S^{(2)} \times\left(S^{(2)}-\mathrm{C}^{(2)}\right)=\nu_{2}$.

For $u, v$ lying in the plane orthogonal to a vector $w$, denote by $\alpha_{w}(u, v)$ the positively oriented angle $(\bmod 2 \pi)$ between $u$ and $v$ (orientation follows the "right-hand rule").

Deprit's action-angle variables $(\Lambda, \Gamma, \Psi, \ell, \gamma, \psi)$ may be, now, defined as follows (compare also Figures 1, 2 and 3).

$$
\begin{aligned}
&\left\{\begin{array}{l}
\Lambda_{i}:=M_{i} \sqrt{\bar{m}_{i} a_{i}} \\
\ell_{i}:=\text { mean anomaly of } x^{(i)} \text { on } \mathfrak{E}_{i}
\end{array}\right. \\
& \begin{cases}\Gamma_{i}:=\left|\mathrm{C}^{(i)}\right|=\Lambda_{i} \sqrt{1-e_{i}^{2}} \\
\gamma_{i}:=\alpha_{\mathrm{C}^{(i)}}\left(\nu_{i}, P_{i}\right)\end{cases} \\
& \Psi_{i}:= \begin{cases}\left|S^{(i+1)}\right| & 1 \leq i \leq n-1 \\
\mathrm{C}_{3}:=\mathrm{C} \cdot k^{(3)} & i=n\end{cases} \\
& \psi_{i}:= \begin{cases}\alpha_{S^{(i+1)}}\left(\nu_{i+2}, \nu_{i+1}\right) & 1 \leq i \leq n-1 \\
\alpha_{k^{(3)}}\left(k^{(1)}, \bar{\nu}\right) & i=n\end{cases}
\end{aligned}
$$

Notice that Deprit variables are defined on an open set of full measure $\mathcal{P}_{*}^{6 n}$ of the Cartesian phase space $\mathcal{P}^{6 n}:=\mathbb{R}^{3 n} \times \mathbb{R}_{*}^{3 n}$, namely, on the set where $\mathrm{C}^{(i)} \neq 0$,

\footnotetext{
${ }^{3}$ In [5] the definition of the node $\nu_{1}$ has the opposite sign.
} 


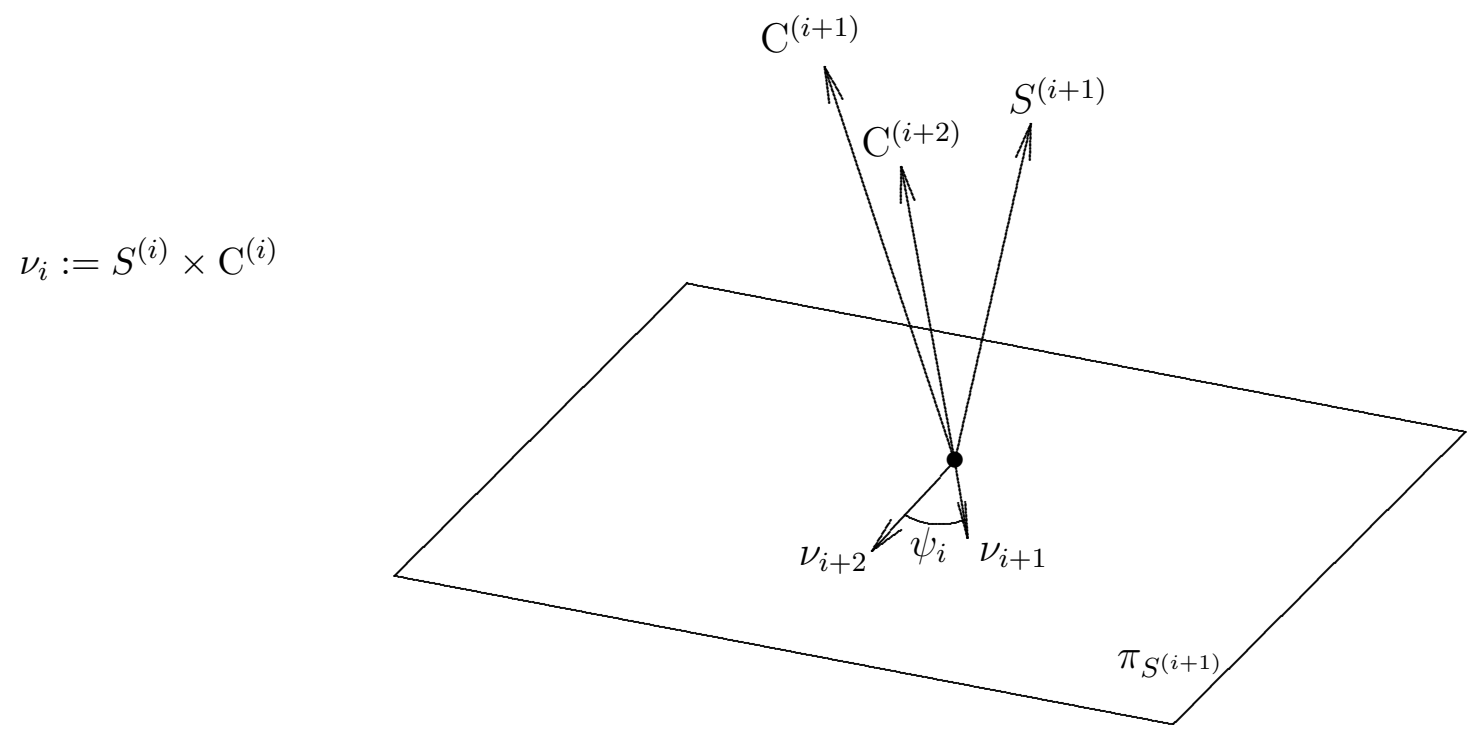

Figure 2: The angle $\psi_{i}$ for $1 \leq i \leq n-2$

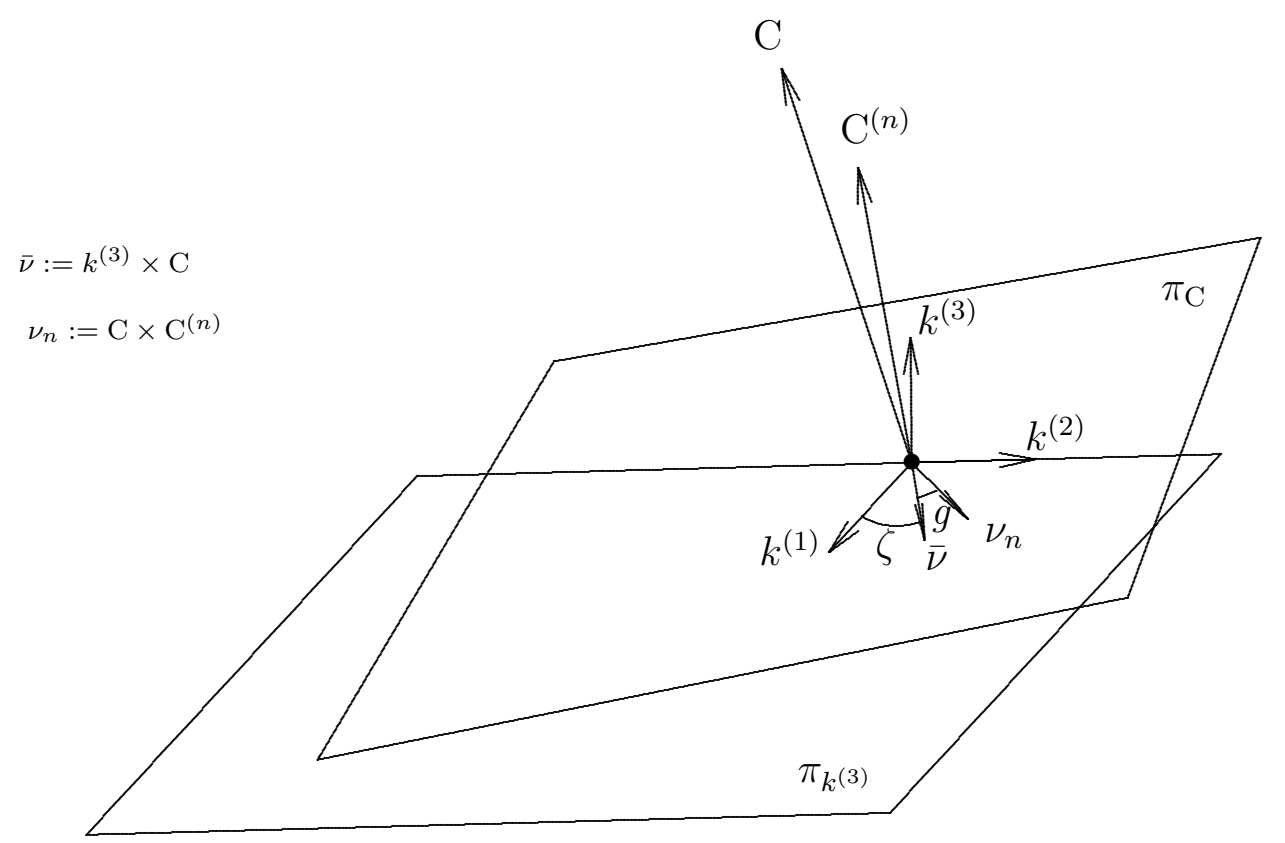

Figure 3: The angles $\psi_{n-1}=: g$ and $\psi_{n}=: \zeta$ 
$e_{i} \in(0,1)$ and the above nodes are well defined. In fact is it not difficult (see [4, Appendix A.1]) to write down inverse formulae showing that the map

$$
\phi_{*}^{(n)}:(y, x) \in \mathcal{P}_{*}^{6 n} \rightarrow(\Lambda, \Gamma, \Psi, \ell, \gamma, \psi) \in \mathbb{R}^{3 n} \times \mathbb{T}^{3 n}
$$

is a real-analytic diffeomorphism from $\mathcal{P}_{*}^{6 n}$ onto its image, namely, the set where ${ }^{4}$

$$
\left\{\begin{array}{l}
0<\Gamma_{i}<\Lambda_{i}, \quad 1 \leq i \leq n, \\
-\Psi_{n-1}<\Psi_{n}<\Psi_{n-1}, \\
\left|\Psi_{i-1}-\Gamma_{i+1}\right|<\Psi_{i}<\Psi_{i-1}+\Gamma_{i+1}, \quad 1 \leq i \leq n-1,
\end{array}\right.
$$

where $\Psi_{0}:=\Gamma_{1}$ is introduced just here.

The main point is the following.

Theorem $(\Lambda, \Gamma, \Psi, \ell, \gamma, \psi)$ are real-analytic symplectic coordinates on $\mathcal{P}_{*}^{6 n}$.

Before giving the proof (in the next section), we make a few remarks.

- The variables $\Psi_{n-1}, \Psi_{n}$ and $\psi_{n}$ (usually denoted also, respectively, $G, \mathrm{C}_{3}$ and $\zeta$ ) are integrals for any rotation invariant Hamiltonian, i.e., for Hamiltonians Poisson-commuting with the three components of the total angular momentum C. Indeed, assigning $G, \mathrm{C}_{3}$ and $\zeta$ corresponds to assign the three components of the total angular momentum $\mathrm{C}$. This fact allows for partial and total symplectic reductions of the phase space of rotation-invariant Hamiltonian systems (compare also next item).

It has to be noted, however, that these action-angle variables, similarly to Delaunay's variables, are particularly suited for the planetary $(1+n)$ body problem, i.e, a system of $(1+n)$ points of masses, $m_{0}, \mu m_{1}, \ldots, \mu m_{n}, \mu$ being, a small number, interacting only through gravity. In such a case if the mass parameter are chosen as

$$
\left\{\begin{array}{c}
M_{i}:=\frac{m_{0} m_{i}}{m_{0}+\mu m_{i}} \\
\bar{m}_{i}:=m_{0}+\mu m_{i}
\end{array}\right.
$$

then the limit $\mu=0$ represents an integrable system ruled by the familiar Keplerian Hamiltonian

$$
-\sum_{i=1}^{n} \frac{M_{i}^{3} \bar{m}_{i}^{2}}{2 \Lambda_{i}^{2}}
$$

describing $n$ decoupled two-body systems formed by the "Sun" $(i=0)$ and the $i^{\text {th }}$ planet with rescaled mass $m_{i}$.

\footnotetext{
${ }^{4}$ Recall that: $\Gamma_{i}=\left|\mathrm{C}^{(i)}\right|=\Lambda_{i} \sqrt{1-} e_{i}^{2} ; \Psi_{n-1}=|C| ; \Psi_{n}:=\mathrm{C}_{3}=\mathrm{C} \cdot k^{(3)} ; \Psi_{i}=\left|S^{(i+1)}\right|=$ $\left|S^{(i)}+\mathrm{C}^{(i+1)}\right|$.
} 
- A special rôle is played by the four pairwise conjugated variables

$$
\left\{\begin{array} { l } 
{ \Psi _ { n - 1 } = | \mathrm { C } | = : G } \\
{ \psi _ { n - 1 } = \alpha _ { \mathrm { C } } ( \overline { \nu } , \nu _ { n } ) = : g }
\end{array} \quad \left\{\begin{array}{l}
\Psi_{n}=\mathrm{C} \cdot k^{(3)}=: \mathrm{C}_{3} \\
\psi_{n}=\alpha_{k^{(3)}}\left(k^{(1)}, \bar{\nu}\right)=: \zeta
\end{array}\right.\right.
$$

Since $G, \mathrm{C}_{3}$ and $\zeta$ are functions of $\mathrm{C}$, they commute with rotational-invariant Hamiltonians. Hence, such Hamiltonians would not depend upon the variables $g$ (conjugated to the integral $G=|\mathrm{C}|$ ) nor the pairwise conjugated integrals $\mathrm{C}_{3}$ and $\zeta$ (but would, in general, depend on $G$ ).

The cyclic angles $g$ and $\zeta$ are directly related to the rotations of the system. In fact,

$$
\zeta \rightarrow \zeta+\alpha, \quad \text { or } \quad g \rightarrow g+\alpha,
$$

correspond to rotations of all the $y^{(i)}$ 's and the $x^{(i)}$ 's by an angle $\alpha$ around, respectively, the $k^{(3)}$-axis or the $\mathrm{C}$-axis. Notice that, differently from $\zeta$, whose motion is trivial (indeed, constant), $g$ is not an integral and it describes the rotation of the node $\nu_{n}$ around the $\mathrm{C}$-axis. However, its motion is known by a trivial quadrature when the remaining motion is integrated.

The fact of having two ignorable angles is what we call reduction.

In general, such reduction lowers the number of degrees of freedom from $3 n$ to $3 n-2$. For three bodies, as well known, the degrees of freedom are 4 .

We stress that such reduction is symplectic, meaning that it is obtained by a ("full dimensional") symplectic transformation of the phase space: this fact is deeper than the usual Jacobi nodes reduction in the three body problem, obtained through restriction to the vertical angular momentum manifold (see below). More precisely, such symplectic reduction "unfolds" the Jacobi reduction, as will be explained in details in the appendix. Indeed, for $n=2$, the variables (4) reduce to two quadruples of $\left(\Lambda_{i}, \Gamma_{i}, \ell_{i}, \gamma_{i}\right)$ 's and to the four variables in (6) where $\mathrm{C}=\mathrm{C}^{(1)}+\mathrm{C}^{(2)}, \bar{\nu}=k^{(3)} \times \mathrm{C}, \nu_{2}=\mathrm{C} \times \mathrm{C}^{(2)}=\mathrm{C}^{(1)} \times \mathrm{C}^{(2)}$. Since the variables $\mathrm{C}_{3}, \zeta, g$, are cyclic, they can be regarded as fixed once and for all in the Hamiltonian. Choosing the values $(G, 0,0)$ physically corresponds to refer the system to a rotating frame having the $k^{(3)}$-axis in the direction of $\mathrm{C}$, the $k^{(1)}$-axis in the direction of the rotating node $\nu_{2}$ and to consider the motion of the remaining 8 variables $\left(\Lambda_{i}, \Gamma_{i}, \ell_{i}, \gamma_{i}\right)$ 's (with $G$ regarded as a parameter). The reduced Hamilton equations correspond to the classical Jacobi's equations.

- The fact that $\mathrm{C}_{3}$ (which is a symplectic action) also disappears in rotationalinvariant Hamiltonians is of deeper nature and is related to having three non commuting integrals, or, equivalently, to the fact that the group of rotations in $\mathbb{R}^{3}$ is not Abelian. We remark, at this respect, that having a cyclic conjugate couple $\left(\mathrm{C}_{3}, \zeta\right)$ into the Hamiltonian has strong consequences on the symplectic structure of the phase space. It is at the basis of a "rotational invariance 
proper degeneracy" which, e.g., prevents direct applications of KAM theories to the $\mathrm{N}$-body problem in the unreduced setting.

- The Theorem is due to A. Deprit even though his setting is slightly different to that described above. More precisely, the original Deprit's reduction goes as follows.

On the instantaneous planes of $\left(y^{(i)}, x^{(i)}\right)$, consider the (planar) polar coordinates

$$
\left\{\begin{array} { l } 
{ R _ { i } : = \frac { y ^ { ( i ) } \cdot x ^ { ( i ) } } { r _ { i } } } \\
{ r _ { i } : = | x ^ { ( i ) } | }
\end{array} \quad \left\{\begin{array}{l}
\Phi_{i}:=\left|\mathrm{C}^{(i)}\right|=\Gamma_{i} \\
\varphi_{i}:=\alpha_{\mathrm{C}^{(i)}}\left(\nu_{i}, x^{(i)}\right)
\end{array}\right.\right.
$$

Notice that the anomaly $\varphi_{i}$ is referred to the node $\nu_{i}$ in (3) as polar axis.

Deprit, then, introduces the variables

$$
(R, \Phi, \Psi, r, \varphi, \psi)
$$

with the $(\Psi, \psi)$ 's as in $(4)$. He proved that such variables are homogeneoussymplectic with respect to the Cartesian variables $(y, x)$, namely, that the 1 -form $y \cdot d x$ is preserved by the transformation $(R, \Phi, \Psi, r, \varphi, \psi) \rightarrow(y, x)$. Now, since the map

$$
\left(R_{i}, \Phi_{i}, r_{i}, \varphi_{i}\right) \rightarrow\left(\Lambda_{i}, \Gamma_{i}, \ell_{i}, \gamma_{i}\right)
$$

is symplectic ${ }^{5}$, the symplecticity of the variables (4) is equivalent to that of the variables (8).

Notice, however, that the map $(\Lambda, \Gamma, \Psi, \ell, \gamma, \psi) \rightarrow(y, x)$ is not homogeneous, because it involves the ("complicated") Kepler map (compare its definition in $\S 2.1)$.

- The proof presented in $\S 2$ below of Deprit's Theorem will be based on two ingredients. The first is the analysis of the three-body case, which reduces to considering a suitable 8-dimensional map $\hat{\phi}^{(2)}$. The second ingredient is a hierarchical (inductive) approach based on a suitable iteration of $\hat{\phi}^{(2)}$, which allows to obtain the variables (4) through a natural inductive construction. Quite informally (the details are in $\S 2.2$ below), the mechanism is the following. At each step (i.e., when the $n^{\text {th }}$ planet is added), the new set of variables is obtained using the formulae relatively to two planets: a fictitious planet of angular momentum equal to $S^{(n-1)}$ (the sum of the angular momenta of the previous $(n-1)$ planets: compare $(2))$ and the new planet, of angular momentum $\mathrm{C}^{(n)}$. The first new case (after the three-body's one) is for $n=3$ (four-body case) and the definition of the new angle variable $\psi_{1}$ appears quite naturally as if it were the "perihelion argument of the fictitious planet".

\footnotetext{
${ }^{5}$ The proof is classical but beware that the node of the anomaly $\varphi_{i}$ is $\nu_{i}$, while in the classical case one would take the fixed node $\bar{\nu}_{i}$ of the orbital plane with the coordinate plane $\left(k^{(1)}, k^{(2)}\right)$.
} 
An analogous inductive construction could be carried out also for the Deprit's set $(R, \Phi, \Psi, r, \varphi, \psi)$ : compare the Remark at the end of $\S 2.2$.

- In [5], Deprit declares that his variables realize a generalization of a set of variables studied by Radau in a paper of 1868 [8], leaving the reader with the feeling that the "full" symplectic reduction in the case $n=2$ was already known since then. But it is not quite so and the generalization Deprit speaks about is only related to the Hamilton equations. Let us briefly discuss this and other historical matters.

- The original spirit of reduction was that of lowering the order of the differential equations governing the motion of two planets around the Sun. This was achieved by Jacobi [6] in 1842, who succeeded by writing a set differential equations of eighth order ${ }^{6}$. As well known, Jacobi's main idea consisted in taking the invariable plane as horizontal plane, so to obtain the opposition relation between the nodes $\bar{\nu}_{i}$ 's of the orbital planes with such plane

$$
\bar{\nu}_{1}+\bar{\nu}_{2} \equiv 0 \text {. }
$$

Such relation causes the lowering of order through the disappearance of nodes from the motion equations.

- In 1868, Radau [8] made such procedure canonical, rewriting the motion equations in Hamiltonian form, in terms of eight canonical variables (the $R_{i}, \Phi_{i}, r_{i}, \varphi_{i}$ above, with $i=1,2$ ). More precisely, Radau treats the Euclidean length of the angular momentum $G=|\mathrm{C}|$ as an external parameter and shows that the node reduction can be performed "before taking derivatives" obtaining an "effective reduced" Hamiltonian governing the three-body motion. Radau himself attributes the procedure to Jacobi ${ }^{7}$. As far as we can say, the original contribution of Radau to the question of the reduction of order in the paper [8] is seemingly related to a different reduction: The construction of canonical variables on the plane of the three bodies, together with a (Jacobi-like) reduction of the node of such plane with the invariable plane. Such different procedure was later extended to $n \geq 2$ by Bennett [1].

- For a long time no one succeeded in extending the Jacobi-Radau reduction technique to more than three bodies. In fact the first non-trivial extension of Jacobi-Radau's method to the four-body case appeared more than a century later, in 1982, and it is due to Françoise Boigey [2]. She introduces a symplectic transformation into the phase space, which realizes a reduction by one degree of freedom using $\mathrm{C}_{3}$ as an action variables (so as to obtain an ignorable coordinate, corresponding

\footnotetext{
${ }^{6} \mathrm{Jacobi}$ wrote a system of seven differential equations, six of them of first order and one of second order.

${ }^{7}$ Quite mysteriously, Whittaker [9] attributes it instead to Radau.
} 
to the anomaly of $\left.\bar{\nu}_{3}\right)$. Next, she introduces a conjugate couple $\left(P_{2}, Q_{2}\right)$ of symplectic variables so that the vertical angular momentum manifold has equation $P_{2}=0=Q_{2}$. In other words, Boigey' couple $\left(P_{2}, Q_{2}\right)$, differently from Deprit's $\left(\mathrm{C}_{3}, \zeta\right)$, is not cyclic. Rather, the origin of $\left(P_{2}, Q_{2}\right)$ is an equilibrium for it (corresponding to vertical angular momentum). This is enough to write the motion equations of the four-body problem as an Hamiltonian system of 7 degrees of freedom. Boigey's basic tools are essentially two: the first, reminiscent of Jacobi's reduction, is the use of two invariant relations coming from the triangular relation $\bar{\nu}_{1}+\bar{\nu}_{2}+\bar{\nu}_{3} \equiv 0$ which generalizes (10), in order to construct the couple $\left(P_{2}, Q_{2}\right)$. The second remarkable ingredient (later used by Deprit) is the use of the action variable $\Psi_{1}=\left|\mathrm{C}^{(1)}+\mathrm{C}^{(2)}\right|$. Boigey also proves that such variable has also some uniqueness features. Finally, to construct its conjugate angle, Boigey introduces a "pseudonode" $N$ which plays the rôle of Deprit's $\nu_{3}$.

- One year later, in 1983, Andre Deprit gave his beautiful and complete picture in the paper [5], which we are revisiting.

\section{Proofs}

In this section we prove Deprit's Theorem by showing that ${ }^{8}$

$$
\Lambda \cdot d \ell+\Gamma \cdot d \gamma+\Psi \cdot d \psi=y \cdot d x+d \chi
$$

where $\chi$ is a real analytic function $\mathrm{on}^{9} \mathcal{P}_{*}^{6 n}$.

Our argument makes explicit use of comparison with Delaunay's variables, which we now recall. Denote, for $1 \leq i \leq n$, by

$$
\bar{\nu}_{i}:=k^{(3)} \times \mathrm{C}^{(i)}
$$

the "Delaunay nodes" and assume that they do not vanish. Then, the Delaunay variables are defined as $(\Lambda, \Gamma, \Theta, \ell, \mathrm{g}, \theta)$, where $\Lambda, \Gamma, \ell$ are as in (4), while

$$
\Theta_{i}:=\mathrm{C}^{(i)} \cdot k^{(3)}, \quad \theta_{i}:=\alpha_{k^{(3)}}\left(k^{(1)}, \bar{\nu}_{i}\right), \quad \mathrm{g}_{i}:=\alpha_{\mathrm{C}^{(i)}}\left(\bar{\nu}_{i}, P_{i}\right) .
$$

In particular, the variables $\Gamma_{i}, \Theta_{i}$ and $\theta_{i}$ uniquely define the angular momenta $\mathrm{C}^{(i)}$ through the formulae

$$
\mathrm{C}^{(i)}\left(\Gamma_{i}, \Theta_{i}, \theta_{i}\right)=\left(\begin{array}{c}
\sqrt{\Gamma_{i}^{2}-\Theta_{i}^{2}} \sin \theta_{i} \\
-\sqrt{\Gamma_{i}^{2}-\Theta_{i}^{2}} \cos \theta_{i} \\
\Theta_{i}
\end{array}\right)
$$

\footnotetext{
${ }^{8}$ As usual, $\Lambda \cdot d \ell+\Gamma \cdot d \gamma+\Psi \cdot d \psi:=\sum_{1 \leq i \leq n}\left(\Lambda_{i} d \ell_{i}+\Gamma_{i} d \gamma_{i}+\Psi_{i} d \psi_{i}\right)$ and $y \cdot d x:=\sum_{1 \leq i \leq n} y^{(i)} \cdot d x^{(i)}$.

${ }^{9}$ Recall the definition of the set $\mathcal{P}_{*}^{6 n}$ before Eq.n (5).
} 


\subsection{Case $n=2$ (three-body case)}

By Eq.s (14) one can express the total angular momentum $\mathrm{C}=\mathrm{C}^{(1)}+\mathrm{C}^{(2)}$ and the nodes $\nu_{1}=\nu_{2}, \bar{\nu}, \bar{\nu}_{1}, \bar{\nu}_{2}$ as functions of $\Gamma, \Theta, \theta$. This, in turn, allows to express the four variables $G, \mathrm{C}_{3}, \zeta, g$ in (6) in terms of $\Gamma, \Theta, \theta$. The Deprit perihelia are instead shifted with respect to Delaunay's:

$$
\gamma_{i}=\alpha_{\mathrm{C}^{(i)}}\left(\nu_{i}, P_{i}\right)=\alpha_{\mathrm{C}^{(i)}}\left(\bar{\nu}_{i}, P_{i}\right)+\alpha_{\mathrm{C}^{(i)}}\left(\nu_{i}, \bar{\nu}_{i}\right)=\mathrm{g}_{i}+\alpha_{\mathrm{C}^{(i)}}\left(\nu_{i}, \bar{\nu}_{i}\right) .
$$

The core of reduction relies upon the eight-dimensional map

$$
(\Gamma, \Theta, g, \theta) \rightarrow(\Gamma, \Psi, \gamma, \psi)
$$

Notice that such map is in turn the symplectic lift of the family of 4-dimensional maps (parametrized by $\Gamma)(\Theta, \theta) \rightarrow(\Psi, \psi)$ which act as the identity on the $\Gamma^{\prime}$ 's variables.

As said before, to avoid overflow of (elementary) computations based on the generating function of the map (16), we prove the symplecticity of (16) indirectly, i.e. , as Deprit, we prefer to use Cartesian variables, namely, we check the symplecticity of the map $\phi_{*}^{(2)}$ in (5). To do this, we shall use the explicit expression of $\phi_{*}^{(2)}$, given in [4], which now we recall, after having fixed some notations.

- We denote by $\mathcal{R}_{1}(i), \mathcal{R}_{3}(\theta)$ the following rotations

$$
\mathcal{R}_{1}(i)=\left(\begin{array}{ccc}
1 & 0 & 0 \\
0 & \cos i & -\sin i \\
0 & \sin i & \cos i
\end{array}\right), \quad \mathcal{R}_{3}(\theta)=\left(\begin{array}{ccc}
\cos \theta & -\sin \theta & 0 \\
\sin \theta & \cos \theta & 0 \\
0 & 0 & 1
\end{array}\right)
$$

- We let $y_{\mathrm{pl}}^{(i)}:=\left(\mathrm{y}_{\mathrm{pl}}^{(i)}, 0\right)$ and $x_{\mathrm{pl}}^{(i)}:=\left(\mathrm{x}_{\mathrm{pl}}^{(i)}, 0\right)$, where

$$
\left(\mathrm{y}_{\mathrm{pl}}^{(i)}, \mathrm{x}_{\mathrm{pl}}^{(i)}\right)=\left(\mathrm{y}_{\mathrm{pl}}^{(i)}\left(\Lambda_{i}, \Gamma_{i}, \ell_{i}, \gamma_{i}\right), \mathrm{x}_{\mathrm{pl}}^{(i)}\left(\Lambda_{i}, \Gamma_{i}, \ell_{i}, \gamma_{i}\right)\right)
$$

is the planar Kepler-map, i.e. : on the Keplerian ellipse with perihelion argument $\gamma_{i}$, eccentricity $e_{i}$ and semimajor axis $a_{i}$ as in $(4), \mathrm{x}_{\mathrm{pl}}^{(i)}=\left(\mathrm{x}_{1}^{(i)}, \mathrm{x}_{2}^{(i)}\right) \in \mathbb{R}^{2}$ is the point of mean anomaly $\ell_{i}$;

$$
\mathrm{y}_{\mathrm{pl}}^{(i)}=\left(\mathrm{y}_{1}^{(i)}, \mathrm{y}_{2}^{(i)}\right):=\beta_{i} \partial_{\ell_{i}} \mathrm{x}_{\mathrm{pl}}^{(i)}:=M_{i} \sqrt{\bar{m}_{i} / a_{i}^{3}} \partial_{\ell_{i}} \mathrm{x}_{\mathrm{pl}}^{(i)}
$$

its conjugate momentum. Indeed, it is well known that

$$
\mathrm{y}_{1}^{(i)} \cdot d \mathrm{x}_{1}^{(i)}+\mathrm{y}_{2}^{(i)} \cdot d \mathrm{x}_{2}^{(i)}=\Lambda_{i} d \ell_{i}+\Gamma_{i} d \gamma_{i}+d \chi_{i}
$$

for some real-analytic function $\chi_{i}$.

- The variables $(\Psi, \psi)$ in $(4)$ will be denoted with the names in (6):

$$
\Psi_{1}=G, \quad \Psi_{2}=\mathrm{C}_{3}, \quad \psi_{1}=g, \quad \psi_{2}=\zeta .
$$


Claim 1 For $n=2$, the map

$$
\phi_{*}^{-1}:(\Lambda, \Gamma, \Psi, \ell, \gamma, \psi) \rightarrow(y, x)
$$

has the following analytical expression:

$$
y^{(i)}=\mathrm{R}_{i} y_{\mathrm{pl}}^{(i)}, \quad x^{(i)}=\mathrm{R}_{i} x_{\mathrm{pl}}^{(i)}, \quad i=1,2,
$$

where $\mathrm{R}_{i} \in \mathrm{SO}(3)$ are the unitary matrices depending upon $\Gamma_{i}, G, \mathrm{C}_{3}, g$ and $\zeta$ given by

$$
\mathrm{R}_{1}=\mathcal{R}_{3}(\zeta) \mathcal{R}_{1}\left(i_{2}^{*}\right) \mathcal{R}_{3}(g) \mathcal{R}_{1}\left(-i_{1}^{*}\right), \quad \mathrm{R}_{2}=\mathcal{R}_{3}(\zeta) \mathcal{R}_{1}\left(i_{2}^{*}\right) \mathcal{R}_{3}(g) \mathcal{R}_{1}\left(i_{2}\right)
$$

with $i_{1}^{*}, i_{2}^{*}, i_{2} \in(0, \pi)$ defined by

$$
\cos i_{1}^{*}=\frac{G^{2}+\Gamma_{1}^{2}-\Gamma_{2}^{2}}{2 G \Gamma_{1}}, \quad \cos i_{2}^{*}=\frac{\mathrm{C}_{3}}{G}, \quad \cos i_{2}=\frac{G^{2}+\Gamma_{2}^{2}-\Gamma_{1}^{2}}{2 G \Gamma_{2}} .
$$

Proof Let $\mathrm{F}=\left(k^{(1)}, k^{(2)}, k^{(3)}\right)$ denote the prefixed orthonormal basis with respect to which $y^{(i)}, x^{(i)}$ split, respectively, as

$$
y^{(i)}=\sum_{j=1}^{3} y_{j}^{(i)} k^{(j)}, \quad x^{(i)}=\sum_{j=1}^{3} x_{j}^{(i)} k^{(j)} .
$$

Consider the three following orthonormal bases. The "invariant basis" $\mathrm{F}_{*}$ formed by the triple $\left(k_{*}^{(1)}, k_{*}^{(2)}, k_{*}^{(3)}\right)$ with $k_{*}^{(1)}$ in the direction of the node $\bar{\nu}$ and $k_{*}^{(3)}$ in the direction of the total angular momentum $\mathrm{C}=\mathrm{C}^{(1)}+\mathrm{C}^{(2)}$ and the "orbital bases" $\mathrm{F}_{1}=\left(k_{1}^{(1)}, k_{1}^{(2)}, k_{1}^{(3)}\right)$ and $\mathrm{F}_{2}=\left(k_{2}^{(1)}, k_{2}^{(2)}, k_{2}^{(3)}\right)$, with $k_{i}^{(1)}$ in the direction of $\nu_{1}=\nu_{2}$ and $k_{i}^{(3)}$ in the direction of $\mathrm{C}^{(i)}$. Here, the nodes $\nu_{1}, \nu_{2}$ and $\bar{\nu}$ are as in (3) (with $n=2)$. Let us consider similar splittings $y_{*}^{(j)}, y_{\mathrm{pl}}^{(j)}$ and $x_{*}^{(j)}, x_{\mathrm{pl}}^{(j)}$ of $y^{(j)}, x^{(j)}$ with respect to $\mathrm{F}_{*}$ and $\mathrm{F}_{j}$. Notice that the expression of $y_{\mathrm{pl}}^{(j)}, x_{\mathrm{pl}}^{(j)}$ in terms of $\Lambda_{j}, \Gamma_{j}$, $\ell_{j}$ and $\gamma_{j}$ is just the one claimed at the beginning of this section, as it follows from the definitions of these variables. Furthermore, from the definition of $\bar{\nu}$ and $\zeta$, there follows that $z^{(j)}=\mathcal{R}_{3}(\zeta) \mathcal{R}_{1}\left(i_{2}^{*}\right) z_{*}^{(j)}$, where $z$ denotes either $y$ or $x$, and $i_{2}^{*}$ is the (convex) angle between $k^{(3)}$ and C. Similarly, from the definition of $\nu_{1}=\nu_{2}$ and $g$ there easily follows that $z_{*}^{(1)}=\mathcal{R}_{3}(g) \mathcal{R}_{1}\left(-i_{1}^{*}\right) z_{\mathrm{pl}}^{(1)}$ and $z_{*}^{(2)}=\mathcal{R}_{3}(g) \mathcal{R}_{1}\left(i_{2}\right) z_{\mathrm{pl}}^{(2)}$, where $i_{1}^{*}, i_{2}$ are the angles of $\mathrm{C}^{(1)}$ and $\mathrm{C}$ and of $\mathrm{C}^{(2)}$ and $\mathrm{C}$. Considering the triangle spanned by $\mathrm{C}^{(1)}, \mathrm{C}^{(2)}$ and $\mathrm{C}$ and the definitions of $G, \mathrm{C}_{3}, \Gamma_{1}$ and $\Gamma_{2}$, one finds the expressions of $i_{1}^{*}, i_{2}^{*}$ and $i_{2}$ as in (21). The claim then follows.

To check (11), we shall use the following simple fact

\section{Claim 2 Let}

$$
x=\mathcal{R}_{3}(\theta) \mathcal{R}_{1}(i) \bar{x}, \quad y=\mathcal{R}_{3}(\theta) \mathcal{R}_{1}(i) \bar{y}, \quad \mathrm{C}:=x \times y, \quad \overline{\mathrm{C}}:=\bar{x} \times \bar{y},
$$


with $x, \bar{x}, y, \bar{y} \in \mathbb{R}^{3}$. Then,

$$
y \cdot d x=\mathrm{C} \cdot k^{(3)} d \theta+\overline{\mathrm{C}} \cdot k^{(1)} d i+\bar{y} \cdot d \bar{x} .
$$

Proof Since the $\mathcal{R}_{k}$ 's are unitary matrices, denoting $(\cdot)^{\prime}$ the derivative with respect to $\theta$ or $i$ and by $(\cdot)^{T}$ matrix transposition, we find

$$
\begin{aligned}
y \cdot d x & =y \cdot\left(\mathcal{R}_{3}^{\prime} \mathcal{R}_{1} \bar{x} d \theta+\mathcal{R}_{3} \mathcal{R}_{1}^{\prime} \bar{x} d i+\mathcal{R}_{3} \mathcal{R}_{1} d \bar{x}\right) \\
& =y \cdot \mathcal{R}_{3}^{\prime} \mathcal{R}_{3}^{T} x d \theta+\bar{y} \cdot \mathcal{R}_{1}^{T} \mathcal{R}_{1}^{\prime} \bar{x} d i+\bar{y} \cdot d \bar{x}
\end{aligned}
$$

and observing that

$$
\mathcal{R}_{3}^{\prime} \mathcal{R}_{3}^{T}=\left(\begin{array}{ccc}
0 & -1 & 0 \\
1 & 0 & 0 \\
0 & 0 & 0
\end{array}\right) \quad \mathcal{R}_{1}^{T} \mathcal{R}_{1}^{\prime}=\left(\begin{array}{ccc}
0 & 0 & 0 \\
0 & 0 & -1 \\
0 & 1 & 0
\end{array}\right)
$$

the claim follows.

We are now ready to check (11). Notice the following expressions for the angular momenta, easily implied by (19) and well known relations on the planar Kepler map

$$
\mathrm{C}^{(i)}=\mathrm{R}_{i}\left(x_{\mathrm{pl}}^{(i)} \times y_{\mathrm{pl}}^{(i)}\right)=\Gamma_{i} \mathrm{R}_{i} k^{(3)} .
$$

From such expressions, the following formula for the total angular momentum follows

$$
\begin{aligned}
\mathrm{C}=\mathrm{C}^{(1)}+\mathrm{C}^{(2)} & =\Gamma_{1} \mathrm{R}_{1} k^{(3)}+\Gamma_{2} \mathrm{R}_{2} k^{(3)} \\
& =\mathcal{R}_{3}(\zeta) \mathcal{R}_{1}\left(i_{2}^{*}\right) \mathcal{R}_{3}(g)\left(\Gamma_{1} \mathcal{R}_{1}\left(-i_{1}^{*}\right)+\Gamma_{2} \mathcal{R}_{1}\left(i_{2}\right)\right) k^{(3)} \\
& =G \mathcal{R}_{3}(\zeta) \mathcal{R}_{1}\left(i_{2}^{*}\right) k^{(3)}
\end{aligned}
$$

having used $\mathcal{R}_{3}(g)\left(\Gamma_{1} \mathcal{R}_{1}\left(-i_{1}^{*}\right)+\Gamma_{2} \mathcal{R}_{1}\left(i_{2}\right)\right) k^{(3)}=\mathcal{R}_{3}(g) G k^{(3)}=G k^{(3)}$, as it easily follows from (21).

Let

$$
\bar{x}^{(1)}:=\mathcal{R}_{3}(g) \mathcal{R}_{1}\left(-i_{1}^{*}\right) x_{\mathrm{pl}}^{(1)}, \quad \bar{y}^{(1)}:=\mathcal{R}_{3}(g) \mathcal{R}_{1}\left(-i_{1}^{*}\right) y_{\mathrm{pl}}^{(1)}
$$

so as to write (compare (19) and (20))

$$
x^{(1)}=\mathcal{R}_{3}(\zeta) \mathcal{R}_{1}\left(i_{2}^{*}\right) \bar{x}^{(1)}, \quad y^{(1)}=\mathcal{R}_{3}(\zeta) \mathcal{R}_{1}\left(i_{2}^{*}\right) \bar{y}^{(1)} .
$$

Put

$$
\overline{\mathrm{C}}^{(1)}:=\bar{x}^{(1)} \times \bar{y}^{(1)}, \quad \mathrm{C}_{\mathrm{pl}}^{(1)}:=x_{\mathrm{pl}}^{(1)} \times y_{\mathrm{pl}}^{(1)} .
$$


Using Claim 2 twice, one easily finds

$$
\begin{aligned}
y^{(1)} \cdot d x^{(1)}= & \mathrm{C}^{(1)} \cdot k^{(3)} d \zeta+\overline{\mathrm{C}}^{(1)} \cdot k^{(1)} d i_{2}^{*}+\bar{y}^{(1)} \cdot d \bar{x}^{(1)} \\
= & \mathrm{C}^{(1)} \cdot k^{(3)} d \zeta+\overline{\mathrm{C}}^{(1)} \cdot k^{(1)} d i_{2}^{*}+\overline{\mathrm{C}}^{(1)} \cdot k^{(3)} d g+\mathrm{C}_{\mathrm{pl}}^{(1)} \cdot k^{(1)} d\left(-i_{1}^{*}\right) \\
& +y_{\mathrm{pl}}^{(1)} \cdot d x_{\mathrm{pl}}^{(1)} \\
= & \mathrm{C}^{(1)} \cdot k^{(3)} d \zeta+\mathrm{C}^{(1)} \cdot e^{(1)} d i_{2}^{*}+\mathrm{C}^{(1)} \cdot e^{(3)} d g+y_{\mathrm{pl}}^{(1)} \cdot d x_{\mathrm{pl}}^{(1)}
\end{aligned}
$$

We have used $\mathrm{C}_{\mathrm{pl}}^{(1)} \cdot k^{(1)}=\Gamma_{1} k^{(3)} \cdot k^{(1)}=0, \mathrm{C}^{(1)}=\mathcal{R}_{3}(\zeta) \mathcal{R}_{1}\left(i_{2}^{*}\right) \overline{\mathrm{C}}^{(1)}$ and we have let

$$
e^{(i)}:=\mathcal{R}_{3}(\zeta) \mathcal{R}_{1}\left(i_{2}^{*}\right) k^{(i)}
$$

With a similar procedure, one finds

$$
y^{(2)} \cdot d x^{(2)}=\mathrm{C}^{(2)} \cdot k^{(3)} d \zeta+\mathrm{C}^{(2)} \cdot e^{(1)} d i_{2}^{*}+\mathrm{C}^{(2)} \cdot e^{(3)} d g+y_{\mathrm{pl}}^{(2)} \cdot d x_{\mathrm{pl}}^{(2)} .
$$

The claim (11) now follows taking the sum of (24) and (26), using (18) and recognizing that

$$
\left\{\begin{array}{l}
k^{(3)} \cdot\left(\mathrm{C}^{(1)}+\mathrm{C}^{(2)}\right)=k^{(3)} \cdot \mathrm{C}=\mathrm{C}_{3} \\
e^{(1)} \cdot\left(\mathrm{C}^{(1)}+\mathrm{C}^{(2)}\right)=e^{(1)} \cdot \mathrm{C}=0 \\
e^{(3)} \cdot\left(\mathrm{C}^{(1)}+\mathrm{C}^{(2)}\right)=e^{(3)} \cdot \mathrm{C}=G
\end{array}\right.
$$

(the first equality is just the definition of $\mathrm{C}_{3}$; for the second and the third equality, use (23) and (25)), with $\chi=\chi_{1}+\chi_{2}$.

\section{$2.2 \quad$ Induction $(n \geq 2)$}

In this section we complete the induction making an explicit use of the Delaunay variables $(\Lambda, \Gamma, \Theta, \ell, g, \theta)$ (compare the definition at the beginning of $\S 2.1$ ). We recall that the map

$$
(\Lambda, \Gamma, \Theta, \ell, \mathrm{g}, \theta) \rightarrow(\Lambda, \Gamma, \Psi, \ell, \gamma, \psi)
$$

is "product map", as the $(4 n)$-dimensional map

$$
\hat{\phi}^{(n)}: \quad(\Gamma, \Theta, \mathrm{g}, \theta) \rightarrow(\Gamma, \Psi, \gamma, \psi)
$$

is independent of ${ }^{10}(\Lambda, \ell)$. Therefore, it is enough to prove that $\hat{\phi}^{(n)}$ is symplectic, provided $\hat{\phi}^{(n-1)}$ is.

The idea of the proof is the following. Assume that $\hat{\phi}^{(n-1)}$ is symplectic and consider the Delaunay coordinates $(\Gamma, \Theta, \mathrm{g}, \theta)$ at rank $n$. Split such coordinates as

$$
\Gamma=\left(\tilde{\Gamma}, \Gamma_{n}\right), \quad \Theta=\left(\tilde{\Theta}, \Theta_{n}\right), \quad \mathrm{g}=\left(\tilde{\mathrm{g}}, \mathrm{g}_{n}\right), \quad \theta=\left(\tilde{\theta}, \theta_{n}\right)
$$

\footnotetext{
${ }^{10}$ This follows from the explicit expression of $\hat{\phi}^{(n)}$ in (28) below. By (14), the angular momenta $\mathrm{C}^{(i)}$ (and hence also their sums $S^{(i)}$ and the nodes $\nu_{i}, \bar{\nu}_{i}$ ) depend only on $\Gamma, \Theta$ and $\theta$.
} 
where $\tilde{\Gamma}, \tilde{\Theta}$, $\tilde{\mathrm{g}}$ and $\tilde{\theta}$ denote the first $(n-1)$ components of $\Gamma, \Theta, \mathrm{g}, \theta$. With the set $(\tilde{\Gamma}, \tilde{\Theta}, \tilde{g}, \tilde{\theta})$, using $\hat{\phi}^{(n-1)}$, construct a set of Deprit variables $(\tilde{\Gamma}, \tilde{\Psi}, \tilde{\gamma}, \tilde{\psi})$ of dimension $4(n-1)$ and leave the variables $\left(\Gamma_{n}, \Theta_{n}, \mathrm{~g}_{n}, \theta_{n}\right)$ unvaried. In particular, the variables $\tilde{\Psi}_{n-2}$ and $\tilde{\Psi}_{n-1}$ will be related to the angular momentum $S^{(n-1)}$ of $(n-1)$ particles by

$$
\tilde{\Psi}_{n-2}=\left|S^{(n-1)}\right|, \quad \tilde{\Psi}_{n-1}=S^{(n-1)} \cdot k^{(3)} ;
$$

the angle $\tilde{\psi}_{n-1}$ will correspond to the longitude of the node of $S^{(n-1)}$ with the $\left(k^{(1)}, k^{(2)}\right)-$ plane ${ }^{11}$. Namely, $\tilde{\Psi}_{n-2}, \tilde{\Psi}_{n-1}$ and $\tilde{\psi}_{n-1}$ can be regarded as three of the Delaunay coordinates of a fictitious body whose perihelion argument is $\tilde{\psi}_{n-2}$ (the angle conjugated to $\tilde{\Psi}_{n-2}$ ). The idea is now to use the formulae (16) of the threebody case, with $\left(\Gamma_{1}, \Theta_{1}, \mathrm{~g}_{1}, \theta_{1}\right)$ and $\left(\Gamma_{2}, \Theta_{2}, \mathrm{~g}_{2}, \theta_{2}\right)$ replaced by the two following quadruples of coordinates: the quadruple $\left(\tilde{\Psi}_{n-2}, \tilde{\Psi}_{n-1}, \tilde{\psi}_{n-2}, \tilde{\psi}_{n-1}\right)$ and the quadruple $\left(\Gamma_{n}, \Theta_{n}, g_{n}, \theta_{n}\right)$, left unvaried at the previous step. This will define the right variables at rank $n$, which, by construction, will turn to be symplectic.

The details are as follows. Let us begin with the analytical expression of $\hat{\phi}^{(n)}$, which is

$$
\begin{aligned}
\gamma_{i} & =\mathrm{g}_{i}+\alpha_{\mathrm{C}^{(i)}}\left(\nu_{i}, \bar{\nu}_{i}\right), \\
\Psi_{i} & = \begin{cases}\left|S^{(i+1)}\right| & i \neq n \\
\Theta_{1}+\cdots+\Theta_{n} & i=n\end{cases} \\
\psi_{i} & = \begin{cases}\alpha_{S^{(i+1)}}\left(\nu_{i+2}, \nu_{i+1}\right) & i \neq n \\
\alpha_{k^{(3)}}\left(k^{(1)}, \nu_{n+1}\right) & i=n\end{cases}
\end{aligned}
$$

It is therefore enough to check that

$$
\Gamma \cdot d \mathrm{~g}+\Theta \cdot d \theta=\Gamma \cdot d \gamma+\Psi \cdot d \psi+d \chi^{(n)}
$$

on $^{12}$ on the set $\mathcal{D}^{4 n}$ of $((\Gamma, \Theta),(\mathrm{g}, \theta)) \in\left(\mathbb{R}_{+}^{n} \times \mathbb{R}^{n}\right) \times \mathbb{T}^{2 n}$ where

$$
\left|\Theta_{i}\right|<\Gamma_{i}, \quad \nu_{i+1} \neq 0, \quad \forall 1 \leq i \leq n
$$

Such inequalities imply that nor the nodes $\bar{\nu}_{i}=k^{(3)} \times \mathrm{C}^{(i)}$ nor the vectors $\mathrm{C}^{(i)}$ or $S^{(i+1)}$ vanish, so that equations (28) are well put.

Assume, inductively, that (29) holds up to $n-1 \geq 2$. Let us define an auxiliary symplectic map $\tilde{\phi}=\tilde{\phi}^{(n)}$. Denote by $\mathcal{D}_{*}^{4 n} \subset \mathcal{D}^{4 n}$ the set where $k^{(3)} \times S^{(n-1)} \neq 0$. If $(\Gamma, \Theta, \mathrm{g}, \theta) \in \mathcal{D}_{*}^{4 n}$, define $(\tilde{\Gamma}, \tilde{\Theta}, \tilde{\mathrm{g}}, \tilde{\theta})$ as in $(27)$. Since

\footnotetext{
${ }^{11}$ Compare the definition (4), with $(n-1)$ replacing $n,(\tilde{\Gamma}, \tilde{\Psi}, \tilde{\gamma}, \tilde{\psi})$ replacing $(\Gamma, \Psi, \gamma, \psi)$.

${ }^{12}$ The restriction $\left|\Theta_{i}\right|<\Gamma_{i}$ is needed to use Delaunay variables, but, regarding the Deprit variables in terms of the Cartesian variables as in (4), the map $\phi_{*}^{(n)}$ in (5) remains symplectic on the larger set $\mathcal{P}_{*}^{6 n}$, defined in $\S 1$. Notice in fact that the subset $\mathcal{P}_{* *}^{6 n}$ of $\mathcal{P}_{*}^{6 n}$ where (30) holds is dense in $\mathcal{P}_{*}^{6 n}$ and that $\phi_{*}^{(n)}$ is regular on $\mathcal{P}_{*}^{6 n}$.
} 
$k^{(3)} \times S^{(n-1)} \neq 0$, the point $(\tilde{\Gamma}, \tilde{\Theta}, \tilde{g}, \tilde{\theta})$ lies on the domain $\mathcal{D}^{4(n-1)}$ of $\hat{\phi}^{(n-1)}$; let $(\tilde{\Gamma}, \tilde{\Psi}, \tilde{\gamma}, \tilde{\psi}) \in \mathbb{R}^{2(n-1)} \times \mathbb{T}^{2(n-1)}$ the image

$$
(\tilde{\Gamma}, \tilde{\Psi}, \tilde{\gamma}, \tilde{\psi})=\hat{\phi}^{(n-1)}(\tilde{\Gamma}, \tilde{\Theta}, \tilde{\mathrm{g}}, \tilde{\theta})
$$

Define then

$$
\tilde{\phi}: \quad(\Gamma, \Theta, g, \theta) \in \mathcal{D}_{*}^{4 n} \rightarrow\left(\left(\tilde{\Gamma}, \Gamma_{n}\right),\left(\tilde{\Psi}, \Theta_{n}\right),\left(\tilde{\gamma}, \mathrm{g}_{n}\right),\left(\tilde{\psi}, \theta_{n}\right)\right) .
$$

By the inductive assumption, $\hat{\phi}^{(n-1)}$ verifies, on $\mathcal{D}^{4(n-1)}$,

$$
\tilde{\Gamma} \cdot d \tilde{\mathrm{g}}+\tilde{\Theta} \cdot d \tilde{\theta}=\tilde{\Gamma} \cdot d \tilde{\gamma}+\tilde{\Psi} \cdot d \tilde{\psi}+d \chi^{(n-1)}
$$

for some $\chi^{(n-1)}$. Hence $\tilde{\phi}$ verifies, on $\mathcal{D}_{*}^{4 n}$

$$
\Gamma \cdot d \mathrm{~g}+\Theta \cdot d \theta=\tilde{\Gamma} \cdot d \tilde{\gamma}+\tilde{\Psi} \cdot d \tilde{\psi}+\Gamma_{n} d g_{n}+\Theta_{n} d \theta_{n}+d \chi^{(n-1)}
$$

Being $S^{(n-1)}$ not parallel to the $k^{(3)}$-axis, there follows that $\left|\tilde{\Psi}_{n-1}\right|<\tilde{\Psi}_{n-2}$. From $(\Gamma, \Theta, g, \theta) \in \mathcal{D}^{4 n}$ there also follows that

$$
\begin{aligned}
& \left|\Theta_{n}\right|<\Gamma_{n} \\
& \left(S^{(n-1)}+\mathrm{C}^{(n)}\right) \times \mathrm{C}^{(n)}=S^{(n)} \times \mathrm{C}^{(n)}=: N_{2} \neq 0 \\
& k^{(3)} \times\left(S^{(n-1)}+\mathrm{C}^{(n)}\right)=k^{(3)} \times S^{(n)}=: \bar{N} \neq 0 .
\end{aligned}
$$

From such formulae one has that

$$
\left(\left(\tilde{\Psi}_{n-2}, \Gamma_{n}\right),\left(\tilde{\Psi}_{n-1}, \Theta_{n}\right),\left(\tilde{\psi}_{n-2}, \mathrm{~g}_{n}\right),\left(\tilde{\psi}_{n-1}, \theta_{n}\right)\right) \in \mathcal{D}^{8}
$$

where $\mathcal{D}^{8}$ is the domain of $\hat{\phi}^{(2)}$.

Let $\left(\left(\tilde{\Gamma}, \Gamma_{n}\right),\left(\tilde{\Psi}, \Theta_{n}\right),\left(\tilde{\gamma}, \mathrm{g}_{n}\right),\left(\tilde{\psi}, \theta_{n}\right)\right)$ be any point for which $(34)$ holds. On such set of points, consider the following transformation $\check{\phi}$. Denote by

$$
\left(\left(\check{\Psi}_{n-2}, \check{\Gamma}_{n}\right),\left(\check{\Psi}_{n-1}, \check{\Psi}_{n}\right),\left(\check{\psi}_{n-2}, \check{\gamma}_{n}\right),\left(\check{\psi}_{n-1}, \check{\psi}_{n}\right)\right)
$$

the image of

$$
\left(\left(\tilde{\Psi}_{n-2}, \Gamma_{n}\right),\left(\tilde{\Psi}_{n-1}, \Theta_{n}\right),\left(\tilde{\psi}_{n-2}, \mathrm{~g}_{n}\right),\left(\tilde{\psi}_{n-1}, \theta_{n}\right)\right)
$$

under $\hat{\phi}^{(2)}$. Such image has the following analytical expression. Let $\mathcal{C}^{(1)}, \mathcal{C}^{(2)}$ be the angular momenta defined as in (14) but with the following substitutions:

$$
\begin{array}{cl}
\left(\Gamma_{1}, \Theta_{1}, \theta_{1}\right) & \rightarrow\left(\Psi_{n-2}, \tilde{\Psi}_{n-1}, \tilde{\psi}_{n-1}\right) \\
\left(\Gamma_{2}, \Theta_{2}, \theta_{2}\right) & \rightarrow\left(\Gamma_{n}, \Theta_{n}, \theta_{n}\right) \\
\mathrm{C} & \rightarrow \mathcal{C}^{(1)}+\mathcal{C}^{(2)}=: \sigma^{(2)} \\
\nu_{1}=\nu_{2} & \rightarrow \sigma^{(2)} \times \mathcal{C}^{(2)}=\mathcal{C}^{(1)} \times \mathcal{C}^{(2)}=: N_{1}=N_{2} \\
\bar{\nu}_{i} & \rightarrow k^{(3)} \times \mathcal{C}^{(i)}=: \bar{N}_{i} \\
\bar{\nu} & \rightarrow k^{(3)} \times \sigma^{(2)}=: \bar{N}
\end{array}
$$


Then, $\hat{\phi}^{(2)}$ has the expression $\check{\Psi}_{n-2}:=\tilde{\Psi}_{n-2}, \check{\Gamma}_{n}:=\Gamma_{n}$ and $^{13}$

$$
\begin{array}{r}
\left\{\begin{array}{l}
\check{\psi}_{n-2}=\tilde{\psi}_{n-2}+\alpha_{\mathcal{C}^{(1)}}\left(N_{1}, \bar{N}_{1}\right) \\
\check{\gamma}_{n}=g_{n}+\alpha_{\mathcal{C}^{(2)}}\left(N_{2}, \bar{N}_{2}\right)
\end{array}\right. \\
\check{\Psi}_{i}= \begin{cases}\left|\sigma^{(2)}\right| & i=n-1 \\
\tilde{\Psi}_{n-1}+\Theta_{n} & i=n\end{cases} \\
\check{\psi}_{i}= \begin{cases}\alpha_{\sigma^{(2)}}\left(\bar{N}, N_{2}\right) & i=n-1 \\
\alpha_{k^{(3)}}\left(k^{(1)}, \bar{N}\right) & i=n\end{cases}
\end{array}
$$

Notice that from $\S 2.1$ (compare the observation at the beginning of the section), one has that the map $\hat{\phi}^{(2)}$ verifies

$$
\sum_{i=n-2}^{n-1} \tilde{\Psi}_{i} d \tilde{\psi}_{i}+\Gamma_{n} d g_{n}+\Theta_{n} d \theta_{n}=\sum_{i=n-2}^{n} \check{\Psi}_{i} d \check{\psi}_{i}+\check{\Gamma}_{n} d \check{\gamma}_{n}+d \hat{\chi}^{(2)}
$$

for some $\hat{\chi}^{(2)}$. Define then $\check{\phi}$ by

$$
\begin{gathered}
\check{\phi}: \quad\left(\left(\tilde{\Gamma}, \Gamma_{n}\right),\left(\tilde{\Psi}, \Theta_{n}\right),\left(\tilde{\gamma}, g_{n}\right),\left(\tilde{\psi}, \theta_{n}\right)\right) \rightarrow \\
\left(\left(\tilde{\Gamma}, \check{\Gamma}_{n}\right),\left(\tilde{\Psi}_{1}, \cdots, \tilde{\Psi}_{n-3}, \check{\Psi}_{n-2}, \check{\Psi}_{n-1}, \check{\Psi}_{n}\right),\left(\tilde{\gamma}, \check{\gamma}_{n}\right),\left(\tilde{\psi}_{1}, \cdots, \tilde{\psi}_{n-3}, \check{\psi}_{n-2}, \check{\psi}_{n-1}, \check{\psi}_{n}\right)\right) .
\end{gathered}
$$

Since $\check{\phi}$ leaves the variables $\tilde{\Gamma}, \tilde{\gamma}, \tilde{\Psi}_{1}, \cdots, \tilde{\Psi}_{n-3}, \tilde{\psi}_{1}, \cdots, \tilde{\psi}_{n-3}$ unvaried, from (38), one has this map verifies

$$
\tilde{\Psi} \cdot d \tilde{\psi}+\tilde{\Gamma} \cdot d \tilde{\gamma}+\Gamma_{n} d g_{n}+\Theta_{n} d \theta_{n}=\sum_{i=1}^{n-3} \tilde{\Psi}_{i} d \tilde{\psi}_{i}+\sum_{i=n-2}^{n} \check{\Psi}_{i} d \check{\psi}_{i}+\tilde{\Gamma} \cdot d \tilde{\gamma}+\check{\Gamma}_{n} d \check{\gamma}_{n}+d \hat{\chi}^{(2)}
$$

Consider now the composition $\check{\phi} \circ \tilde{\phi}$, which sends a point $((\Gamma, \Theta),(\mathrm{g}, \theta)) \in \mathcal{D}_{*}^{4 n}$ to the point defined by the right hand side of (38). From Eq.s (33) and (39) there follows that $\check{\phi} \circ \tilde{\phi}$ verifies

$$
\Gamma \cdot d \mathrm{~g}+\Theta \cdot d \theta=\sum_{i=1}^{n-3} \tilde{\Psi}_{i} d \tilde{\psi}_{i}+\sum_{i=n-2}^{n} \check{\Psi}_{i} d \check{\psi}_{i}+\tilde{\Gamma} \cdot d \tilde{\gamma}+\check{\Gamma}_{n} d \check{\gamma}_{n}+d \chi^{(n)}
$$

with $\chi^{(n)}:=\chi^{(n-1)}+\hat{\chi}^{(2)}$. The proof will be finished as soon as one recognizes that $^{14} \check{\phi} \circ \tilde{\phi}$ coincides with the map $\hat{\phi}^{(n)}$ in $(28)$.

\footnotetext{
${ }^{13}$ Compare (6) and (15) with $\check{\psi}_{n-2}, \check{\gamma}_{n}, \check{\Psi}_{n-1}, \check{\Psi}_{n}, \check{\psi}_{n-1}, \check{\psi}_{n}$ replacing, respectively, $\gamma_{1}, \gamma_{2}, G$, $\mathrm{C}_{3}, g, \zeta$.

${ }^{14}$ Again, since $\hat{\phi}_{*}^{(n)}$ is regular on $\hat{\mathcal{D}}^{4 n}$ and $\hat{\mathcal{D}}_{*}^{4 n}$ is dense in $\hat{\mathcal{D}}^{4 n}$, being $\hat{\phi}_{*}^{(n)}$ symplectic on $\hat{\mathcal{D}}_{*}^{4 n}$ we get the thesis on the larger set $\mathcal{D}^{4 n}$.
} 
We limit ourselves to check that the angle $\check{\psi}_{n-2}$ in (38) coincides with the angle $\psi_{n-2}$ in (28) (the other checks being trivial).

Let us first notice that the angle $\tilde{\psi}_{n-2}$ in (32) is given by

$$
\tilde{\psi}_{n-2}=\alpha_{S^{(n-1)}}\left(\tilde{N}, \tilde{N}_{n-1}\right)=\alpha_{S^{(n-1)}}\left(\tilde{N}, \nu_{n-1}\right)
$$

where $\tilde{N}:=k^{(3)} \times S^{(n-1)}$ and $\tilde{N}_{n-1}:=S^{(n-1)} \times \mathrm{C}^{(n-1)}=\nu_{n-1}$. Now, in (35), one has the following identifications $\mathcal{C}^{(1)}:=S^{(n-1)}, \mathcal{C}^{(2)}:=\mathrm{C}^{(n)}, \sigma^{(2)}=S^{(n)}, N_{1}=$ $\sigma^{(2)} \times \mathcal{C}^{(2)}=S^{(n)} \times \mathrm{C}^{(n)}=\nu_{n}, \bar{N}_{1}=k^{(3)} \times \mathcal{C}^{(1)}=k^{(3)} \times S^{(n-1)}=\tilde{N}$. Therefore, from (36) and (40),

$$
\begin{aligned}
\check{\psi}_{n-2} & =\tilde{\psi}_{n-2}+\alpha_{\mathcal{C}^{(1)}}\left(N_{1}, \bar{N}_{1}\right) \\
& =\alpha_{S^{(n-1)}}\left(\tilde{N}, \nu_{n-1}\right)+\alpha_{S^{(n-1)}}\left(\nu_{n}, \tilde{N}\right) \\
& =\alpha_{S^{(n-1)}}\left(\nu_{n}, \nu_{n-1}\right)=\psi_{n-2}
\end{aligned}
$$

Remark Let $(R, \Phi, \Psi, r, \varphi, \psi)$ the variables defined in $(7) \div(8)$ and let $(R, \Phi$, $\Theta, r, \mathrm{f}, \theta)$ be defined as follows. The variables $(R, r, \Phi)$ are in common between the two sets; $(\Theta, \theta)$ are just the Delaunay's (13); while the anomalies $f_{i}$ are referred with respect to the Delaunay nodes $\bar{\nu}_{i}$ in $(12)$ :

$$
\mathrm{f}_{i}=\alpha_{\mathrm{C}(i)}\left(\bar{\nu}_{i}, x^{(i)}\right)
$$

It is very easy to realize that such set of variables is symplectic and that the analytical dependence of the $(\Phi, \Psi, \varphi, \psi)$ 's with respect to the $(\Phi, \Theta, f, \theta)$ 's is just the same as that of the $(\Gamma, \Psi, \gamma, \psi)$ 's with respect to the $(\Gamma, \Theta, \mathrm{g}, \theta)$ 's (described at the beginning of $\S 2.1$ ). Thus, the previous inductive construction can be equally applied to construct the $(R, \Phi, \Psi, r, \varphi, \psi)$ 's, starting from the $(R, \Phi, \Theta, r, \mathrm{f}, \theta)$ 's.

\section{A Deprit's energy-time variables}

Some of the variables in (4) loose their meaning out of the domain $\mathcal{P}_{*}^{6 n}$, i.e., when some eccentricity $e_{i}$ vanishes or $e_{i} \geq 1$ : in the former case, the variables $(\ell, \gamma)$ are not defined, in the latter case $(\Lambda, \ell)$ are not.

Even though Deprit mentioned that an important application of his variables would have been to use them in connection with the planetary model, he underlined quite clearly that his node reduction could apply more generally to any rotation invariant system. Indeed, the map $(y, x) \rightarrow(R, \Phi, \Psi, r, \varphi, \psi)$ is actually well defined (i.e., injective) on the larger subset $\overline{\mathcal{P}}^{6 n} \supset \mathcal{P}_{*}^{6 n}$ of the phase space $\mathcal{P}^{6 n}=\mathbb{R}^{6 n} \times \mathbb{R}_{*}^{3 n}$ simply defined by inequalities

$$
\overline{\mathcal{P}}^{6 n}: \quad \nu_{i} \neq 0
$$

where $\nu_{i}$ are the nodes (3). 
The purpose of this appendix is to show that also the approach presented in this paper can be extended to an open set of full measure of the global phase space, namely, to the set

$$
\overline{\mathcal{P}}_{*}^{6 n}: \quad y^{(i)} \cdot x^{(i)} \neq 0, \quad \nu_{i} \neq 0 .
$$

Indeed, we will show how to construct "energy-time" variables $(E, \bar{\Gamma}, \bar{\Psi}, \tau, \bar{\gamma}, \bar{\psi})$, which can be defined on the set $\overline{\mathcal{P}}_{*}^{6 n}$ so that the symplectic proof of $\S 2$ goes over essentially unchanged.

Consider the Two-Body Hamiltonian (1). Inequalities $\nu_{i}=S^{(i)} \times \mathrm{C}^{(i)} \neq 0$ in particular imply that $\mathrm{C}^{(i)}=x^{(i)} \times y^{(i)} \neq 0$. Then, the motion generated by $h_{i}$ starting from an initial datum $(y, x) \in \overline{\mathcal{P}}_{*}^{6 n}$ evolves on a Keplerian conics $\mathfrak{E}_{i}$ whose parameter $p_{i}$ and eccentricity $e_{i}$ are respectively given by

$$
p_{i}=\frac{\left|\mathrm{C}^{(i)}\right|^{2}}{M_{i}^{2} \bar{m}_{i}} \quad e_{i}=\sqrt{\frac{2}{M_{i}}} \frac{\left|\mathrm{C}^{(i)}\right|}{M_{i} \bar{m}_{i}} \sqrt{h_{i}-\bar{E}_{i}}
$$

where

$$
\bar{E}_{i}=-\frac{M_{i}\left(M_{i} \bar{m}_{i}\right)^{2}}{2\left|\mathrm{C}^{(i)}\right|^{2}} .
$$

Such conics turn out to have positive eccentricity provided $y^{(i)} \cdot x^{(i)} \neq 0$. Indeeed, splitting the linear momenta $y^{(i)}$ as

$$
y^{(i)}=R_{i} \frac{x^{(i)}}{r_{i}}-\frac{\mathrm{C}^{(i)} \times x^{(i)}}{r_{i}^{2}}
$$

where $r_{i}:=\left|x^{(i)}\right|$ and $R_{i}:=\frac{y^{(i)} x^{(i)}}{\left|x^{(i)}\right|}$, one easily rewrites $h_{i}$ as

$$
h_{i}=\frac{R_{i}^{2}}{2 M_{i}}+\left(\frac{\left|\mathrm{C}^{(i)}\right|}{\sqrt{2 M_{i}} r_{i}}-\frac{\sqrt{2 M_{i}}\left(M_{i} \bar{m}_{i}\right)}{2\left|\mathrm{C}^{(i)}\right|}\right)^{2}+\bar{E}_{i}
$$

which, combined with (42), shows that $e_{i}>0$ when $R_{i}=\frac{y^{(i)} \cdot x^{(i)}}{\left|x^{(i)}\right|} \neq 0$.

Let then $P_{i}$ the perihelion of $\mathfrak{E}_{i}, \mathcal{A}_{i}$ the area spanned from $P_{i}$ to the point $x^{(i)}$. Define $\bar{\Gamma}, \bar{\Psi}, \bar{\gamma}, \bar{\psi}$ simply generalizing the respective definitions of $\Gamma, \Psi, \gamma, \psi$ in (4) and put

$$
E_{i}:=h_{i} \quad \tau_{i}:=\frac{2 M_{i} \mathcal{A}_{i}}{\left|\mathrm{C}^{(i)}\right|} .
$$

On $\mathcal{P}_{*}^{6 n}$, i.e., in correspondence of eccentricities verifying $0<e_{i}<1$, the variables $\bar{\Gamma}, \bar{\Psi}, \bar{\gamma}, \bar{\psi}$ trivially coincide with the variables $\Gamma, \Psi, \gamma, \psi$ in $(4)$; the variables $(E, \tau)$ are symplectically related to $(\Lambda, \ell)$ through

$$
E_{i}=-\frac{\bar{m}_{i}^{2} M_{i}^{3}}{2 \Lambda_{i}^{2}} \quad \tau_{i}=\frac{\Lambda_{i}^{3}}{\bar{m}_{i}^{2} M_{i}^{3}} \bar{\ell}_{i}
$$

where $\bar{\ell}_{i}$ is the lifting of $\ell_{i}$ over $\mathbb{R}$. 
Notice that $E$ and $\tau$ have the physical dimension of energy and time (whence the name of this appendix).

The map $\bar{\phi}_{*}^{(n)}$ which assigns to a point $(y, x) \in \overline{\mathcal{P}}_{*}^{6 n}$ the variables $(E, \bar{\Gamma}, \bar{\Psi}, \tau, \bar{\gamma}, \bar{\psi})$ is well defined (i.e., real-analytic and injective) onto its image, i.e., the subset of $(E, \bar{\Gamma}, \bar{\Psi}, \tau, \bar{\gamma}, \bar{\psi}) \in \mathbb{R}^{3 n} \times\left(\mathbb{R}^{n} \times \mathbb{T}^{2 n}\right)$ where $\bar{\Gamma}_{i}>0$ and

$$
\left\{\begin{array}{l}
E_{i}>-\frac{M_{i}\left(M_{i} \bar{m}_{i}\right)^{2}}{2 \bar{\Gamma}_{i}^{2}}, \quad 1 \leq i \leq n, \\
-\bar{\Psi}_{n-1}<\bar{\Psi}_{n}<\bar{\Psi}_{n-1}, \\
\left|\bar{\Psi}_{i-1}-\bar{\Gamma}_{i+1}\right|<\bar{\Psi}_{i}<\bar{\Psi}_{i-1}+\bar{\Gamma}_{i+1}, \quad 1 \leq i \leq n-1 .
\end{array}\right.
$$

At this point the inductive proof of $\S 2$ adapts immediately to the present variables (as, in fact, the $(\Lambda, \lambda)$ or the $(E, \tau)$ variables do not play any rôle in such a proof).

\section{B Unfolding of Jacobi's reduction of the nodes}

The variables (4) "unfold" Jacobi's reduction of the nodes [6] for the three-body problem. This can be seen by considering the expressions of the Delaunay's variables (13) in terms of the Deprit variables (4).

The expression of the angular momenta (22) in terms of $\Gamma_{i}, G, \mathrm{C}_{3}, \zeta$ and $g$ allows to define the nodes $\bar{\nu}, \bar{\nu}_{i}$ and $\nu_{i}$, which gives the inversion formulae for the angles $\mathrm{g}_{i}$ :

$$
\mathrm{g}_{i}=\gamma_{i}+\alpha_{\mathrm{C}^{(i)}}\left(\bar{\nu}_{i}, \nu_{i}\right), \quad(i=1,2) .
$$

Next, recalling the definition of $\psi_{2}=\zeta$ in (4) one finds

$$
\theta_{i}=\alpha_{k^{(3)}}\left(k^{(1)}, \bar{\nu}_{i}\right)=\alpha_{k^{(3)}}\left(k^{(1)}, \bar{\nu}\right)+\alpha_{k^{(3)}}\left(\bar{\nu}, \bar{\nu}_{i}\right)=\zeta+\alpha_{k^{(3)}}\left(\bar{\nu}, \bar{\nu}_{i}\right),
$$

giving the inversion formulae for the $\theta_{i}$ 's. Finally, identifying $\Theta_{1}, \Theta_{2}$ with the third components of $\mathrm{C}^{(1)}, \mathrm{C}^{(2)}$ in (22), one finds

$$
\left\{\begin{array}{l}
\Theta_{1}=\frac{\mathrm{C}_{3}}{2}+\frac{\mathrm{C}_{3}}{2 G^{2}}\left(\Gamma_{1}^{2}-\Gamma_{2}^{2}\right)-\frac{\sqrt{\left(G^{2}-\mathrm{C}_{3}^{2}\right)\left(\Gamma_{1}^{2}-\left(\Gamma_{2}-G\right)^{2}\right)\left(\left(\Gamma_{2}+G\right)^{2}-\Gamma_{1}^{2}\right)}}{2 G^{2}} \cos g \\
\Theta_{2}=\frac{\mathrm{C}_{3}}{2}-\frac{\mathrm{C}_{3}}{2 G^{2}}\left(\Gamma_{1}^{2}-\Gamma_{2}^{2}\right)+\frac{\sqrt{\left(G^{2}-\mathrm{C}_{3}^{2}\right)\left(\Gamma_{1}^{2}-\left(\Gamma_{2}-G\right)^{2}\right)\left(\left(\Gamma_{2}+G\right)^{2}-\Gamma_{1}^{2}\right)}}{2 G^{2}} \cos g
\end{array}\right.
$$

completing the inversion formulae.

Jacobi's reduction of the nodes is obtained in the vertical angular momentum submanifold, i.e., in $\left\{\mathrm{C}_{1}=\mathrm{C}_{2}=0\right\}=\left\{\mathrm{C} \| k^{(3)}\right\}$. On such (symplectic) submanifold the node $\bar{\nu}=k^{(3)} \times \mathrm{C}$ vanishes and the angles $g=\alpha_{\mathrm{C}}\left(\bar{\nu}, \nu_{2}\right)$ and $\zeta=\alpha_{k^{(3)}}\left(k^{(1)}, \bar{\nu}\right)$ loose their meaning. However, in the limit $\mathrm{C}_{3} \rightarrow \mathrm{C}$ or, equivalently, $\mathrm{C} \rightarrow G k^{(3)}$ one has

$$
\zeta+g:=\alpha_{k^{(3)}}\left(k^{(1)}, \bar{\nu}\right)+\alpha_{\mathrm{C}}\left(\bar{\nu}, \nu_{2}\right) \rightarrow \alpha_{k^{(3)}}\left(k^{(1)}, \bar{\nu}\right)+\alpha_{k^{(3)}}\left(\bar{\nu}, \nu_{2}\right)=\alpha_{k^{(3)}}\left(k^{(1)}, \nu_{2}\right) .
$$


This, suggest to define the angle

$$
\bar{g}:=\alpha_{k^{(3)}}\left(k^{(1)}, \nu_{2}\right)
$$

in the vertical submanifold $\left\{\mathrm{C}_{1}=\mathrm{C}_{2}=0\right\}$. Then, since, in the $n=2$ case, it is

$\nu_{1}=\nu_{2}=\mathrm{C}^{(1)} \times \mathrm{C}^{(2)}=\left(\mathrm{C}^{(1)}+\mathrm{C}^{(2)}\right) \times \mathrm{C}^{(2)}=\mathrm{C} \times \mathrm{C}^{(2)}=G k^{(3)} \times \mathrm{C}^{(2)}=G \bar{\nu}_{2}=-G \bar{\nu}_{1}$

from (45) there follows

$$
\begin{aligned}
& \theta_{2}=\alpha_{k^{(3)}}\left(k^{(1)}, \bar{\nu}_{2}\right)=\alpha_{k^{(3)}}\left(k^{(1)}, \nu_{2}\right)=\bar{g} \\
& \theta_{1}=\alpha_{k^{(3)}}\left(k^{(1)}, \bar{\nu}_{1}\right)=\alpha_{k^{(3)}}\left(k^{(1)},-\nu_{2}\right)=\bar{g}+\pi \text { ("opposition of the nodes") }
\end{aligned}
$$

Furthermore, since, in such limit, $\alpha_{\mathrm{C}^{(i)}}\left(\bar{\nu}_{i}, \nu_{i}\right)=0$, then, $\mathrm{g}_{i}=\gamma_{i}$ and (46) becomes

$$
\left\{\begin{array}{l}
\Theta_{1}=\frac{G}{2}+\frac{1}{2 G}\left(\Gamma_{1}^{2}-\Gamma_{2}^{2}\right) \\
\Theta_{2}=\frac{G}{2}-\frac{1}{2 G}\left(\Gamma_{1}^{2}-\Gamma_{2}^{2}\right)
\end{array}\right.
$$

which, together with the relations already discussed, i.e., $\mathrm{g}_{i}=\gamma_{i}$ and

$$
\left\{\begin{array}{l}
\theta_{1}=\bar{g}+\pi \\
\theta_{2}=\bar{g}
\end{array} \quad i=1,2,\right.
$$

are recognized as the classical formulae for Jacobi's reduction of the nodes, apart from an inessential shift by $\bar{g}$ in the definition of $\theta_{i}(\bar{g}$ is cyclic and in Jacobi's reduction is usually taken to be zero).

\section{References}

[1] T. L. Bennett. On the reduction of the problem of $n$ bodies. Messenger math., XXXIV:113-120, 1905.

[2] F. Boigey. Élimination des nouds dans le problème newtonien des quatre corps. Celestial Mech., 27(4):399-414, 1982.

[3] L. Chierchia and G. Pinzari. Planetary Birkhoff normal forms, 2010, Preprint. http://www.mat.uniroma3.it/users/chierchia/WWW/english_version. html\#preprints.

[4] L. Chierchia and G. Pinzari. The planetary n-body problem: Symplectic foliation, reductions and invariant tori, 2010, Preprint. http://www.mat.uniroma3.it/ users/chierchia/WWW/english_version.html\#preprints.

[5] A. Deprit. Elimination of the nodes in problems of $n$ bodies. Celestial Mech., 30(2):181-195, 1983. 
[6] C. G. J. Jacobi. Sur l'élimination des noeuds dans le problème des trois corps. Astronomische Nachrichten, Bd XX:81-102, 1842.

[7] F. Malige, P. Robutel, and J. Laskar. Partial reduction in the $n$-body planetary problem using the angular momentum integral. Celestial Mech. Dynam. Astronom., 84(3):283-316, 2002.

[8] R. Radau. Sur une transformation des équations différentielles de la dynamique. Ann. Sci. Ec. Norm. Sup., 5:311-375, 1868.

[9] E. T. Whittaker. A treatise on the analytical dynamics of particles and rigid bodies: With an introduction to the problem of three bodies. 4th ed. Cambridge University Press, New York, 1959. 